\title{
On Chromatic Polynomial of Elementary h-Uniform Hyper Cycles
}

\author{
Abdul Jalil M. Khalaf and Mahdi Gareep Sabbar \\ Department of Mathematics, Faculty of Computer Science and Mathematics, University of Kufa, \\ P.O. Box 21, Najaf, Iraq \\ abduljaleel.khalaf@uokufa.edu.iq
}

\begin{abstract}
One of the most popular and useful areas of graph theory is graph colorings. Frequently, we are concerned with determining the least number of colors with which we can achieve a proper coloring on a hypergraph. Furthermore, we want to count the possible number of different proper colorings on a hygraph with a given number of colors which is called the chromatic polynomial.
\end{abstract}

Key words: Graph theory, graph colorings, hygraph, polynomial, chromatic, h-uniform, hypercycle

\section{INTRODUCTION}

Let $\mathrm{P}(\mathrm{G}, \lambda)$ denote the chromatic polynomial of a graph G. Two graphs $\mathrm{G}$ and $\mathrm{H}$ are chromatically equivalent, written $\mathrm{G} \sim \mathrm{H}$, if $\mathrm{P}(\mathrm{G}, \lambda)=\mathrm{P}(\mathrm{H}, \lambda)$. A graph $\mathrm{G}$ is chromatically unique, written $\mathrm{X}$-unique, if for any graph $\mathrm{H}$, $\mathrm{G} \sim \mathrm{H}$ implies that $\mathrm{G}$ is isomorphic with $\mathrm{H}$. Many papers have been published about chromaticity of graphs which study the chromatically equivalent and the chromatically unique of graphs, see, for example (Khalaf and Peng, 2009, 2010). For more details about chromatic polynomials and chromaticity of graphs, we refer to the monograph (Dong et al., 2005). Hypergraph theory is a part of general study of combinatorial properties of finite families of finite sets. A hypergraph is defined to be a family of hypergraphes which are sets of vertices of cardinality not necessarily 2 (as for graphs).

The concept of a hypergraph was first introduced by C. Berge at Tihany colloquium in 1960 to extend, simplify and unify the results a bout graph and he developed many properties of vertex coloring of a hypergraph (Berge, 1973, 1989; Barchadt and Lazuka, 2007).

\section{MATERIALS AND METHODS}

Definition 1.1; Berge (1973, 1989): The couple $H=(V$, $\varepsilon)$ where $\left\{v_{1}, v_{2}, \ldots, v_{n}\right\}(n \in N)$ is a finite set of vertices. And $\varepsilon(\mathrm{H})=\left\{\mathrm{e}_{1}, \mathrm{e}_{2}, \ldots, \mathrm{e}_{\mathrm{m}}\right\}(\mathrm{m} \in \mathrm{N})$ is an edge-sets with $\mathrm{e}_{\mathrm{i}} \subseteq \mathrm{V}$ $(\mathrm{H})$ and $\left|\mathrm{e}_{\mathrm{i}}\right| \geq 1(1 \leq \mathrm{i} \leq \mathrm{m})$, then we say that $\mathrm{H}$ is a hypergraph of order $|\mathrm{V}|=\mathrm{n}$ and size $|\varepsilon|=\mathrm{m}$ where $\mathrm{e}_{\mathrm{i}}$ is called an edge or hyperedge.

Definition 1.2; Zhao (2009): Let $H=(V, \varepsilon)$ be a hypergraph. If no hyperedge of $\varepsilon(\mathrm{H})$ is subset of any other hyperedge of $\varepsilon(\mathrm{H})$, then we say that $\mathrm{H}$ is sperner.
Definition 1.3; Allagan (2014): Let $H=(V, \varepsilon)$ be a hypergraph then we say that $\mathrm{H}$ is a simple hypergraph, if all hyperedges are differents.

Definition 1.4; Borowieceki and Ewa (2000): Let $H=(V$, $\varepsilon)$ be a hypergraph of size $m \in N$ and Let $e_{i}, e_{j} \in \varepsilon(H)(1 \leq i$, $\mathrm{j} \leq \mathrm{m})$, if $\mathrm{e}_{\mathrm{i}} \cap \mathrm{e}_{\mathrm{j}}=\phi$ or $\mathrm{e}_{\mathrm{i}} \cap \mathrm{e}_{\mathrm{j}}=\{\mathrm{v}\}$ where, $v \in \mathrm{V}(\mathrm{H})$ then we say that $\mathrm{H}$ is a linear hypergraph.

Definition 1.5; Walter (2009): Let $H=(V, \varepsilon)$ be a hypergraph of size $m \in N$ and Let $e_{i} \epsilon \varepsilon(H)(1 \leq i \leq m)$ be a hyperedge if $\left|e_{i}\right|=h$ for each $i$, then we say that $H$ is h-uniform hypergraph, notice that 2-uniform hypergraph is just a graph. The number of hyperedges containing a vertex $v \in V(H)$ is its degree $d_{H}(v)$.

Definition 1.6: Let $H=(V, \varepsilon)$ be a hypergraph and let $\mathrm{u}$, $v \in \mathrm{V}(\mathrm{H})$ where $\mathrm{u} \neq \mathrm{v}$, then $\mathrm{u}, \mathrm{v}$ are lying in the same component, if there are vertices $\mathrm{u}=\mathrm{v}_{0}, \mathrm{v}_{1}, \ldots, \mathrm{v}_{\mathrm{k}}=v \in \mathrm{V}(\mathrm{H})$ and hyperedges $\mathrm{e}_{1}, \ldots, \mathrm{e}_{\mathrm{k}} \in \boldsymbol{\varepsilon}(\mathrm{H})$ such that $v_{\mathrm{i}-1}, v_{\mathrm{i}} \in \mathrm{e}_{\mathrm{i}}$ for each $\mathrm{i}(1 \leq \mathrm{i} \leq \mathrm{k})$. Thus, if $\mathrm{H}$ has only one component then $\mathrm{H}$ is connected, otherwise $\mathrm{H}$ has $\mathrm{r} \geq 2 \quad(\mathrm{r} \in \mathrm{N})$ connected components.

Definition 1.7; West (2005): Let $H=(V, \varepsilon)$ be a hypergraph. A hyperpath (or path) of length $\mathrm{L}$ joining two vertices $\mathrm{u}$ and $\mathrm{v}$ in $\mathrm{H}$ is a subhypergraph consisting of $\mathrm{L}+1$ distinct vertices $\mathrm{u}=\mathrm{v}_{0}, \mathrm{v}_{1}, \ldots, \mathrm{v}_{\mathrm{L}}=\mathrm{v}$ and $\mathrm{L}$ distinct hyperedges $\mathrm{e}_{1}, \mathrm{e}_{2}, \ldots, \mathrm{e}_{\mathrm{L}}$ of and such that $v_{\mathrm{j}-1}, v_{\mathrm{j}} \in \mathrm{e}_{\mathrm{j}}$ for each $\mathrm{j}(1 \leq \mathrm{j} \leq \mathrm{L})$. An example is given in Fig. 1 .

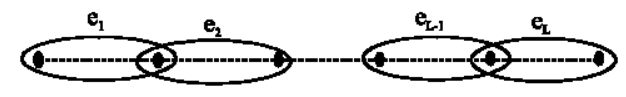

Fig. 1: A hyperpath

Corresponding Author: Abdul Jalil M. Khalaf, Department of Mathematics, Faculty of Computer Science and Mathematics, University of Kufa, P.O. Box 21, Najaf, Iraq, abduljaleel.khalaf@uokufa.edu.iq 


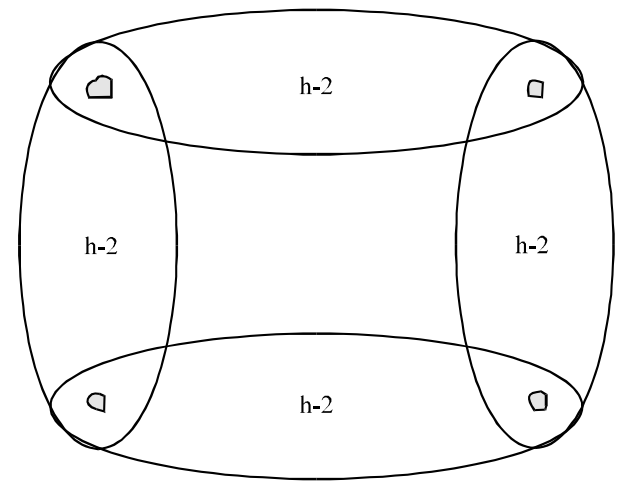

Fig. 2: $\mathrm{C}_{4}^{\mathrm{h}}$ an elementary hypercycle

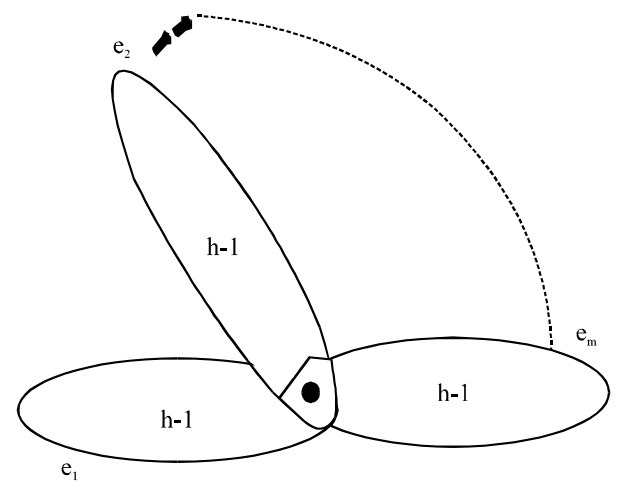

Fig. 3: A hyperstar

Definition 1.8: Let $H=(V, \varepsilon)$ be a hypergraph. A hypercycle (cycle) $\mathrm{C}$ of Length $\mathrm{L}$ in $\mathrm{H}$ is a subhypergraph connecting $\mathrm{L}$ different vertices $v_{1}, \ldots, v_{\mathrm{L}}$ and $\mathrm{L}$ different hyperedges $\mathrm{e}_{1}, \mathrm{e}_{2}, \ldots, \mathrm{e}_{\mathrm{L}}$ such that $v_{\mathrm{j}}, v_{\mathrm{j}+1} \in \mathrm{e}_{\mathrm{j}}$ for each $\mathrm{j}(1 \leq \mathrm{j} \leq \mathrm{L}-1)$ and $v_{1}, v_{\mathrm{L}} \in \mathrm{e}_{\mathrm{L}}$.

If $d_{c}\left(v_{j}\right)=2$ for each $j$ and $d_{c}(y)=1$ for each other vertex $y \in \cup_{j=1}^{L} e_{j}$, then we say that $C$ is an elementary hypercycle. An elementary h-uniform hypercycle with $\mathrm{m} \geq 3$ is denoted by $\mathrm{C}_{\mathrm{m}}^{\mathrm{h}}$ and with 2 hyperedges by $\mathrm{C}_{2}^{\mathrm{h}}$ or $\mathrm{C}(2, \mathrm{~h})$. If we join the end vertices of an hyperedge, then we get a hypercycle of length 1 denoted by $\mathrm{C}_{1}^{\mathrm{h}}$. Elementary hypercycle $\mathrm{C}_{4}^{\mathrm{h}}$ is shown in Fig. 2.

Definition 1.9; Donmen (1993): Let $H=(V, \varepsilon)$ be a connected h-uniform hypergraph without hypercycles. Then, $\mathrm{H}$ is called h-uniform hypertree and denoted $\mathrm{T}_{\mathrm{m}}^{\mathrm{h}}$ where, $m$ is the number of its hyperedges. If all hyperedge of $\mathrm{T}_{\mathrm{m}}^{\mathrm{h}}$ are intersecting in only one vertex then we say that $\mathrm{T}_{\mathrm{m}}^{\mathrm{h}}$ is a hyperstar as shown in Fig. 3 .

Definition 1.10; Borowiecki and Lazuka (2000) and Drgas-Burchardt and Lazuka (2006): Let $\mathrm{H}=(\mathrm{V}(\mathrm{H})$ $(\mathrm{V}, \varepsilon)$ ) be a hypergraph and $\lambda \in \mathrm{N}$, a $\lambda$-coloring of $\mathrm{H}$ is a function $\mathrm{f:} V(\mathrm{H}) \rightarrow\{1, \ldots, \lambda\}$ such that for each $e \in \varepsilon(\mathrm{H})$, here exists $x, y$ in $e$ for which $f(x) \neq f(y)$.

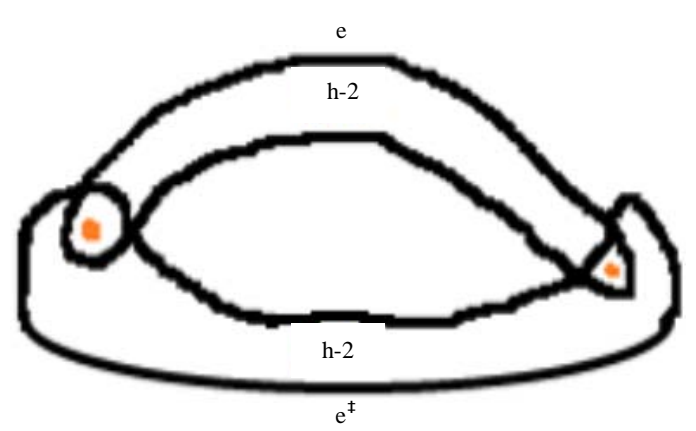

Fig. 4: $\mathrm{e}^{*}$ inactive hyperedge

The number of different $\lambda$-coloring of $\mathrm{H}$ is given by a polynomial $\mathrm{P}(\mathrm{H}, \lambda)$ of degree $|\mathrm{V}(\mathrm{H})|$ in $\lambda$ called the chromatic polynomial of $\mathrm{H}$.

Definition 1.11; Barchadt and Lazuka (2007): Two hypergraphs $\mathrm{H}_{1}$ and $\mathrm{H}_{2}$ are said to be chromatically equivalent, denoted by $\left(\mathrm{H}_{1} \sim \mathrm{H}_{2}\right)$, if $\mathrm{P}\left(\mathrm{H}_{1}, \lambda\right)=\left(\mathrm{H}_{2}, \lambda\right)$. A simple hypergraph $\mathrm{H}_{1}$ is chromatically unique, if $\mathrm{H}_{1} \cong \mathrm{H}_{2}$ for every simple hypergraph $\mathrm{H}_{2}$ such that $\mathrm{H}_{1} \sim \mathrm{H}_{2}$; that is the structure of $\mathrm{H}_{1}$ is uniquely determined up to isomorphism by its chromatic polynomial.

Definition 1.12; Barchadt and Lazuka (2007): Let $H$ be

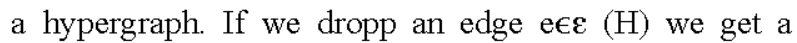
hypergraph $\mathrm{H}^{-}$which is chromatically equivalent to $\mathrm{H}$, then $\mathrm{e}$ is called chromatically inactive. Otherwise, e is said to be chromatically active. The edge $e^{*}$ in Fig. 4 is chromatically inactive $\mathrm{H}$.

\section{RESULTS AND DISCUSSION}

Some known results on enumeration of $P(H, \lambda)$ Theorem 2.1; Borowiecki and Lazuka (2000): Let $H$ be a hypergraph with order $n$ and size $m$, then:

$$
\mathrm{P}(\mathrm{H}, \lambda)=\lambda^{\mathrm{n}}+\mathrm{a}_{\mathrm{n}-1} \lambda^{\mathrm{n}-1}+, \ldots, \mathrm{a}_{2} \lambda^{2}+\mathrm{a}_{1} \lambda
$$

where, $\mathrm{a}_{\mathrm{i}}=\Sigma_{\mathrm{i} z 0}(-1)^{\mathrm{j}} \mathrm{N}(\mathrm{i}, \mathrm{j})$ for $1 \leq \mathrm{i} \leq \mathrm{n}-1$ and $\mathrm{N}(\mathrm{i}, \mathrm{j})$ denoted the number of subhypergraphs of $\mathrm{H}$ with $|\mathrm{V}(\mathrm{H})|$ vertices, $i$ components and $j$ edges.

Theorem 2.2; Kashif (2011): Let $H=(V, \varepsilon)$ be a hypergraph and let $\mathrm{H}$-e be the hypergraph obtained by deleting some edge e $\epsilon \varepsilon(\mathrm{H})$, H.e be the hypergraph obtained by contracting all vertices in e to a common vertex $\mathrm{v}$ and dropping all chromatically inactive edges then:

$$
\mathrm{P}(\mathrm{H}, \lambda)=\mathrm{P}(\mathrm{H}-\mathrm{e}, \lambda)-\mathrm{P}(\mathrm{H} . \mathrm{e}, \lambda)
$$




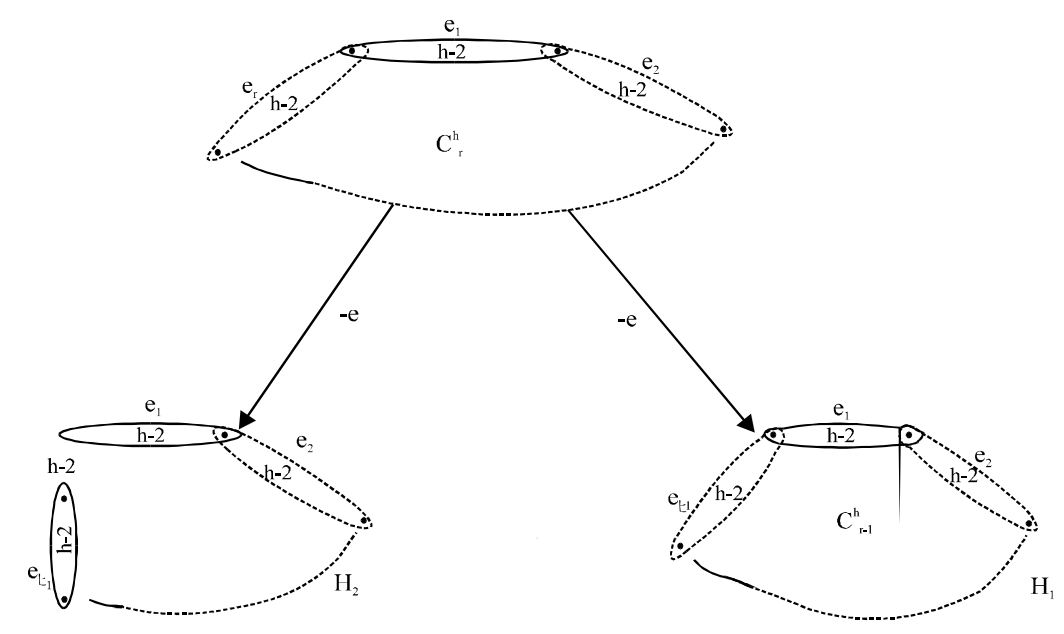

Fig. 5: Hypercycle

Theorem 2.3; West (2005): Assume that $H_{1}, H_{2}, \ldots, H_{m}$ be a connected components of hypergraph $\mathrm{H}$, then:

$$
\mathrm{P}(\mathrm{H}, \lambda)=\prod_{\mathrm{i}=1}^{\mathrm{m}} \mathrm{P}\left(\mathrm{H}_{\mathrm{i}}, \lambda\right)
$$

Theorem 2.4; Bokhary et al. (2009): Let $H$ be a hypergraph such that:

Where:

$$
\mathrm{H}=\mathrm{H}_{1} \mathrm{UH}_{2}, \ldots, \mathrm{UH}_{\mathrm{m}} \text { for } \mathrm{m} \geq 2
$$

$$
\mathrm{H}_{\mathrm{i}} \cap \mathrm{H}_{\mathrm{j}}=\mathrm{k}_{\mathrm{p}}, \mathrm{i} \neq \mathrm{j} \text { and } \bigcap_{\mathrm{i}=1}^{\mathrm{m}} \mathrm{H}_{\mathrm{i}}=\mathrm{k}_{\mathrm{p}}
$$

where, $k_{p}$ is a complete graph with $P \geq 1$ vertices, then:

$$
P(H, \lambda)=\left[P\left(k_{p}, \lambda\right)\right]^{1 \cdot m} \prod_{i=1}^{m} P\left(H_{i}, \lambda\right)
$$

Theorem 2.5; Borowiecki and Ewa (2007): Let $\mathrm{T}_{\mathrm{m}}^{\mathrm{h}}$ be a linear h-uniform hypertree with size $\mathrm{m}$ and $\mathrm{h} \geq 2$ then:

$$
P\left(T_{m}^{h}, \lambda\right)=\lambda\left(\lambda^{h-1}-1\right)^{m}
$$

Theorem 2.6; Tomescu (1998): Let $C_{m}^{h}$ is elementary h-uniform hypertree with size $m$ then:

$$
P\left(C_{m}^{h}, \lambda\right)=\left(\lambda^{h-1}-1\right)^{m}+(-1)^{m}(\lambda-1)
$$

Elementary h-uniform hypercycle: In this section, we give the recurrence relation for the chromatic polynomial of an elementary h-uniform hypercycle.

Example 3.1: Let $\mathrm{C}_{3}^{3}, \mathrm{C}_{4}^{3}, \mathrm{C}_{5}^{3}$ be three elementary $\mathrm{h}=$ uniform hypercycles. Apply the theorem (3.3.6) on $\mathrm{C}_{3}^{3}$, $\mathrm{C}_{4}^{3}, \mathrm{C}_{5}^{3}$ resp:

$$
\begin{aligned}
& P\left(C_{3}^{3}, \lambda\right)=\left(\lambda^{2}-1\right)^{3}+(-1)^{3}(\lambda-1)= \\
& \lambda^{6}-3 \lambda^{4}+3 \lambda^{2}-1-(\lambda-1)=\lambda^{6}-3 \lambda^{4}+3 \lambda^{2}-1-\lambda+1= \\
& \lambda^{6}-3 \lambda^{4}+3 \lambda^{2}-\lambda
\end{aligned}
$$

And:

$$
\begin{aligned}
& \mathrm{P}\left(\mathrm{C}_{4}^{3}, \lambda\right)=\left(\lambda^{2}-1\right)^{4}+(-1)^{4}(\lambda-1)= \\
& \lambda^{8}-4 \lambda^{6}+6 \lambda^{4}-4 \lambda^{2}+1+\lambda-1=\lambda^{8}-4 \lambda^{6}+4 \lambda^{4}-4 \lambda^{2}+\lambda
\end{aligned}
$$

Also:

$$
\begin{aligned}
& \mathrm{P}\left(\mathrm{C}_{5}^{3}, \lambda\right)=\left(\lambda^{2}-1\right)^{5}+(-1)^{5}(\lambda-1)= \\
& \lambda^{10}-5 \lambda^{8}+10 \lambda^{6}-10 \lambda^{4}+5 \lambda^{2}-1-\lambda+1= \\
& \lambda^{10}-5 \lambda^{8}+10 \lambda^{6}-10 \lambda^{4}+5 \lambda^{2}-\lambda
\end{aligned}
$$

To introduce the relation, we need this proposition.

Proposition 3.2: For $r \geq 2, h \geq 3$ we have:

$$
P\left(C_{r}^{h}, \lambda\right)=\lambda^{h-1}\left(\lambda^{h-1}-1\right)^{r-1}-P\left(C_{r-1}^{h}, \lambda\right)
$$

Proof: Let $\mathrm{C}_{\mathrm{r}}^{\mathrm{h}}$ be an elementary h-uniform hypercycle as shown in Fig. 5. If we delete any hyperedge and contract it we obtain two hypergraphs $\mathrm{H}_{1}$ and $\mathrm{H}_{2}$, thus by the theorem (2.2) we get:

$$
P\left(C_{r}^{h}, \lambda\right)=P\left(H_{1}, \lambda\right)-P\left(H_{2}, \lambda\right)
$$

where, $H_{1}$ composed from $h-2$ isolated vertices and hypertree $\mathrm{T}_{\mathrm{r}-1,1}^{\mathrm{h}}$, thus, by the theorems (2.3 and 2.5):

$$
\mathrm{P}\left(\mathrm{H}_{1}, \lambda\right)=\lambda^{\mathrm{h}-2} \lambda\left(\lambda^{\mathrm{h}-1}-1\right)^{\mathrm{r}-1}=\lambda^{\mathrm{h}-1}\left(\lambda^{\mathrm{h}-1}-1\right)^{\mathrm{r}-1}
$$

And $\mathrm{H}_{2}$ is just hypercycle $\mathrm{C}_{\mathrm{r}-1}^{\mathrm{h}}$, thus: 


$$
\mathrm{P}\left(\mathrm{H}_{2}, \lambda\right)=\mathrm{P}\left(\mathrm{C}_{\mathrm{r}-1}^{\mathrm{h}}, \lambda\right)
$$

Substitute 5 and 6 in 4 we get:

$$
P\left(C_{r}^{h}, \lambda\right)=\lambda^{h-1}\left(\lambda^{h-1}-1\right)^{r-1}-P\left(C_{r-1}^{h}, \lambda\right)
$$

As required:

Example 3.3: Consider $\mathrm{C}_{3}^{3}, \mathrm{C}_{2}^{3}, \mathrm{C}_{1}^{3}$. By the theorem (2.6):

$$
\begin{aligned}
& C_{3}^{3}=\left(\lambda^{2}-1\right)^{3}-(\lambda-1)=\lambda^{6}-3 \lambda^{4}+3 \lambda^{2}-\lambda \\
& C_{2}^{3}=\left(\lambda^{2}-1\right)^{2}+(\lambda-1)=\lambda^{4}-2 \lambda^{2}+\lambda
\end{aligned}
$$

Now by applying our result (3.2) and the theorem (2.6):

$$
\begin{aligned}
& P\left(C_{3}^{3}, \lambda\right)=\lambda^{2}\left(\lambda^{2}-1\right)^{2}-P\left(C_{2}^{2}, \lambda\right)= \\
& \lambda^{2}\left(\lambda^{4}-2 \lambda^{2}+1\right)-\left[\left(\lambda^{2}-1\right)^{2}+(\lambda-1)\right]=\lambda^{6}-3 \lambda^{4}+3 \lambda^{2}-\lambda
\end{aligned}
$$

Which is equal to 7. Also:

$$
P\left(C_{1}^{3}, \lambda\right)=\left(\lambda^{2}-1\right)-(\lambda-1)=\lambda^{2}-\lambda
$$

Then:

$$
\begin{aligned}
& P\left(C_{2}^{3}, \lambda\right)=\lambda^{2}\left(\lambda^{2}-1\right)-P\left(C_{1}^{3}, \lambda\right)= \\
& \lambda^{4}-\lambda^{2}-\left(\left(\lambda^{2}-1\right)-(\lambda-1)\right)=\lambda^{4}-\lambda^{2}-\left(\lambda^{2}-\lambda\right)=\lambda^{4}-2 \lambda^{2}+\lambda
\end{aligned}
$$

Which is equal to 7. Now, we can introduce the general recurrence formula for any elementary h-uniform hypercycle.

Theorem 3.4: For $r \geq 2, h \geq 3$, the recurrence relation for the chromatic polynomial of an elementary h-uniform hypercycle $\mathrm{C}_{\mathrm{r}}^{\mathrm{h}}$ is given by:

$$
\begin{aligned}
& \mathrm{P}\left(\mathrm{C}_{\mathrm{r}}^{\mathrm{h}}, \lambda\right)=\lambda^{\mathrm{h}-1} \mathrm{P}\left(\mathrm{C}_{\mathrm{r}-1}^{\mathrm{h}}, \lambda\right)-\left(\lambda^{\mathrm{h}-1}-1\right) \mathrm{P}\left(\mathrm{C}_{\mathrm{r}-2}^{\mathrm{h}}, \lambda\right)- \\
& \lambda^{\mathrm{h}-1}(\lambda-1)\left[(-1)^{\mathrm{r}-1}-(-1)^{\mathrm{r}-2}\right]
\end{aligned}
$$

Notice: If $\mathrm{r}$ is odd, then:

$$
P\left(C_{r}^{h}, \lambda\right)=\lambda^{h-1} P\left(C_{r-1}^{h}, \lambda\right)-\left(\lambda^{h-1}-1\right) P\left(C_{r-2}^{h}, \lambda\right)-2 \lambda^{h-1}(\lambda-1)
$$

If $\mathrm{r}$ is even, then:

$$
P\left(C_{r}^{h}, \lambda\right)=\lambda^{h-1} P\left(C_{r-1}^{h}, \lambda\right)-\left(\lambda^{h-1}-1\right) P\left(C_{r-2}^{h}, \lambda\right)-2 \lambda^{h-1}(\lambda-1)
$$

Proof: Let $\mathrm{C}_{\mathrm{r}}^{\mathrm{h}}$ be an elementary h-uniform hypercycle. By applying proposition (3.2) on $\mathrm{C}_{\mathrm{r}}^{\mathrm{h}}$ and $\mathrm{C}_{\mathrm{r}-1}^{\mathrm{h}}$ then we get:

$$
P\left(C_{r}^{h}, \lambda\right)=\lambda^{h-1}\left(\lambda^{h-1}-1\right)^{r-1}-P\left(C_{r-1}^{h}, \lambda\right)
$$

But:

$$
P\left(C_{r-1}^{h}, \lambda\right)=\left[\lambda^{h-1}\left(\lambda^{h-1}-1\right)^{r-2}-P\left(C_{r-2}^{h}, \lambda\right)\right.
$$

Substitute above result we get:

$$
\begin{aligned}
& =\lambda^{\mathrm{h}-1}\left(\lambda^{\mathrm{h}-1}-1\right)^{\mathrm{r}-1}-\left[\lambda^{\mathrm{h}-1}\left(\lambda^{\mathrm{h}-1}-1\right)^{\mathrm{r}-2}-\mathrm{P}\left(\mathrm{C}_{\mathrm{r}-2}^{\mathrm{h}}, \lambda\right)\right]= \\
& \lambda^{\mathrm{h}-1}\left(\lambda^{\mathrm{h}-1}-1\right)^{\mathrm{r}-1}-\lambda^{\mathrm{h}-1}\left(\lambda^{\mathrm{h}-1}-1\right)^{\mathrm{r}-2}+\mathrm{P}\left(\mathrm{C}_{\mathrm{r}-2}^{\mathrm{h}}, \lambda\right)
\end{aligned}
$$

By adding and subtracting the terms $(-1)^{r-1} \lambda^{h-1}(\lambda-1)$ and $(-1)^{r-2} \lambda^{h-1}(\lambda-1)$ we get:

$$
\begin{aligned}
& \mathrm{P}\left(\mathrm{C}_{\mathrm{r}}^{\mathrm{h}}, \lambda\right)=\lambda^{\mathrm{h}-1}\left(\lambda^{\mathrm{h}-1}-1\right)^{\mathrm{r}-1}-\lambda^{\mathrm{h}-1}\left(\lambda^{\mathrm{h}-1}-1\right)^{\mathrm{r}-2}+\mathrm{P}\left(\mathrm{C}_{\mathrm{r}-2}^{\mathrm{h}}, \lambda\right)+ \\
& (-1)^{\mathrm{r}-1} \lambda^{\mathrm{h}-1}(\lambda-1)-(-1)^{\mathrm{r}-1} \lambda^{\mathrm{h}-1}(\lambda-1)+(-1)^{\mathrm{r}-2} \lambda^{\mathrm{h}-1}(\lambda-1)-(-1)^{\mathrm{r}-2} \lambda^{\mathrm{h}-1}(\lambda-1)= \\
& {\left[\lambda^{\mathrm{h}-1}\left(\lambda^{\mathrm{h}-1}-1\right)^{\mathrm{r}-1}+(-1)^{\mathrm{r}-1} \lambda^{\mathrm{h}-1}(\lambda-1)\right]-\left[\lambda^{\mathrm{h}-1}\left(\lambda^{\mathrm{h}-1}-1\right)^{\mathrm{r}-2}+(-1)^{\mathrm{r}-2} \lambda^{\mathrm{h}-1}(\lambda-1)\right]+} \\
& \mathrm{P}\left(\mathrm{C}_{\mathrm{r}-2}^{\mathrm{h}}, \lambda\right)-\left[(-1)^{\mathrm{r}-1} \lambda^{\mathrm{h}-1}(\lambda-1)-(-1)^{\mathrm{r}-2} \lambda^{\mathrm{h}-1}(\lambda-1)\right]= \\
& \lambda^{\mathrm{h}-1}\left[\left(\lambda^{\mathrm{h}-1}-1\right)^{\mathrm{r}-1}+(-1)^{\mathrm{r}-1}(\lambda-1)\right]-\lambda^{\mathrm{h}-1}\left[\left(\lambda^{\mathrm{h}-1}-1\right)^{\mathrm{r}-2}+(-1)^{\mathrm{r}-2}(\lambda-1)\right]+ \\
& \mathrm{P}\left(\mathrm{C}_{\mathrm{r}-2}^{\mathrm{h}}, \lambda\right)-\lambda^{\mathrm{h}-1}(\lambda-1)\left[(-1)^{\mathrm{r}-1}-(-1)^{\mathrm{r}-2}\right]=\lambda^{\mathrm{h}-1} \mathrm{P}\left(\mathrm{C}_{\mathrm{r}-1}^{\mathrm{h}}, \lambda\right)-\lambda^{\mathrm{h}-1} \mathrm{P}\left(\mathrm{C}_{\mathrm{r}-2}^{\mathrm{h}}, \lambda\right)+ \\
& \mathrm{P}\left(\mathrm{C}_{\mathrm{r}-2}^{\mathrm{h}}, \lambda\right)-\lambda^{\mathrm{h}-1}(\lambda-1)\left[(-1)^{\mathrm{r}-1}-(-1)^{\mathrm{r}-2}\right]=\lambda^{\mathrm{h}-1} \mathrm{P}\left(\mathrm{C}_{\mathrm{r}-1}^{\mathrm{h}}, \lambda\right)- \\
& \lambda^{\mathrm{h}-1}(\lambda-1) \mathrm{P}\left(\mathrm{C}_{\mathrm{r}-2}^{\mathrm{h}}, \lambda\right)-\lambda^{\mathrm{h}-1}(\lambda-1)\left[(-1)^{\mathrm{r}-1}-(-1)^{\mathrm{r}-2}\right]
\end{aligned}
$$

As required:

Example 3.5: From example (3.1) we know that:

$$
\begin{gathered}
\mathrm{P}\left(\mathrm{C}_{3}^{3}, \lambda\right)=\lambda^{6}-3 \lambda^{4}+3 \lambda^{2}-\lambda \\
\mathrm{P}\left(\mathrm{C}_{4}^{3}, \lambda\right)=\lambda^{8}-4 \lambda^{6}+6 \lambda^{4}-4 \lambda^{2}+\lambda \\
\mathrm{P}\left(\mathrm{C}_{5}^{3}, \lambda\right)=\lambda^{10}-5 \lambda^{8}+10 \lambda^{6}-10 \lambda^{4}+5 \lambda^{2}-\lambda
\end{gathered}
$$

Now, if we apply our result (3.4) on $\mathrm{C}_{5}^{3}$ we get:

$$
\begin{aligned}
& \mathrm{P}\left(\mathrm{C}_{5}^{3}, \lambda\right)=\lambda^{2} \mathrm{P}\left(\mathrm{C}_{4}^{3}, \lambda\right)-\left(\lambda^{2}-1\right) \mathrm{P}\left(\mathrm{C}_{3}^{3}, \lambda\right)-\lambda^{2}(\lambda-1)\left[(-1)^{4}-(-1)^{3}\right]= \\
& \lambda^{2}\left[\lambda^{8}-4 \lambda^{6}+6 \lambda^{4}-4 \lambda^{2}+\lambda\right]-\left(\lambda^{2}-1\right)\left[\lambda^{6}-3 \lambda^{4}+3 \lambda^{2}-\lambda\right]-2 \lambda^{2}(\lambda-1)= \\
& \lambda^{2}\left[\lambda^{8}-4 \lambda^{6}+6 \lambda^{4}-4 \lambda^{2}+\lambda\right]-\left(\lambda^{2}-1\right)\left[\lambda^{6}-3 \lambda^{4}+3 \lambda^{2}-\lambda\right]-2 \lambda^{2}(\lambda-1)= \\
& \lambda^{10}-4 \lambda^{8}+6 \lambda^{6}-4 \lambda^{4}+\lambda^{3}-\lambda^{8}+3 \lambda^{6}-3 \lambda^{4}+\lambda^{3}+\lambda^{6}-3 \lambda^{4}+3 \lambda^{2}-\lambda-2 \lambda^{3}+ \\
& 2 \lambda^{2}=\lambda^{10}-5 \lambda^{8}+10 \lambda^{6}-10 \lambda^{4}+5 \lambda^{2}-\lambda
\end{aligned}
$$


Which is equal to 12 .

Example 3.6: Consider $\mathrm{C}_{2}^{4}, \mathrm{C}_{3}^{4}, \mathrm{C}_{4}^{4}$. By applying the theorem (2.6):

$$
\begin{gathered}
P\left(C_{2}^{4}, \lambda\right)=\left(\lambda^{3}-1\right)^{2}+(\lambda-1)=\lambda^{6}-2 \lambda^{3}+\lambda \\
P\left(C_{3}^{4}, \lambda\right)=\left(\lambda^{3}-1\right)^{3}+(\lambda-1)=\lambda^{9}-3 \lambda^{6}+3 \lambda^{2}-\lambda \\
P\left(C_{4}^{4}, \lambda\right)=\left(\lambda^{3}-1\right)^{4}+(\lambda-1)=\lambda^{12}-4 \lambda^{9}+6 \lambda^{6}-4 \lambda^{3}+\lambda
\end{gathered}
$$

By applying our result (3.4):

$$
\begin{aligned}
& \mathrm{P}\left(\mathrm{C}_{4}^{4}, \lambda\right)=\lambda^{3} \mathrm{P}\left(\mathrm{C}_{3}^{4}, \lambda\right)-\left(\lambda^{3}-1\right) \mathrm{P}\left(\mathrm{C}_{2}^{4}, \lambda\right)-\lambda^{3}(\lambda-1)\left[(-1)^{3}-(-1)^{2}\right]= \\
& \lambda^{3}\left[\lambda^{9}-3 \lambda^{6}+3 \lambda^{3}-\lambda\right]-\left(\lambda^{3}-1\right)\left[\lambda^{6}-2 \lambda^{3}+\lambda\right]+2 \lambda^{4}-2 \lambda^{3}=\lambda^{12}-3 \lambda^{9}+3 \lambda^{6}-\lambda^{4}+ \\
& 2 \lambda^{6}-\lambda^{4}+\lambda^{6}-2 \lambda^{3}+\lambda+2 \lambda^{4}-2 \lambda^{3}=\lambda^{12}-4 \lambda^{9}+6 \lambda^{6}-4 \lambda^{3}+\lambda
\end{aligned}
$$

Which is equal to 15 .

\section{CONCLUSION}

In this study we introduce the recurrence relation for the chromatic polynomial of an elementary h-uniform hypercycle.

\section{REFERENCES}

Allagan, J.A., 2014. Chromatic polynomials of some (m, 1)-Hyperwheels. Comput. Sci. J. Moldova, 22: 21-36.

Barchadt, E.O. and E. Lazuka, 2007. On chromatic polynomials of hypergraph. Appl. Math. Lehers, 20: $1250-1254$.

Berge, C., 1973. Graphs and Hypergraphs. 2nd Edn., North-Holland Publishing Company, Amsterdam, Netherlands, ISBN:9780444103994, Pages: 528.

Berge, C., 1989. Hypergraphs: Combinatorics of Finite Sets. Elsevier Publishing Company, New York, USA., ISBN:9780444874894, Pages: 254.
Bokhary, S.A., I. Tomescu and A.A. Bhatti, 2009. On the chromaticity of multi-bridge hypergraphs. Graphs Comb., 25: 145-152.

Borowiecki, M. and E. Lazuka, 2000. Chromatic polynomials of hypergraphs. Discussiones Mathematicae Graph Theory, 20: 293-301.

Borowiecki, M. and L. Ewa, 2007. On cyclomaticity of hypergraphs. Discrete Math., 307: 1418-1429.

Borowiecki, M.L. and E. Lazuka, 2000. Chromatic polynomials of hypergraphs. Discussiones Mathematicae Graph Theor., 20: 293-301.

Dong, F.M., K.M. Koh and K.L. Teo, 2005. Chromatic Polynomials and Chromaticity of Graphs. World Scientific Publishing Company, Singapore, ISBN:9789812563170, Pages: 357.

Donmen, K., 1993. Chromatic polynome von graphen and hypergraphn. Ph.D Thesis, University of Dusseldorf, Düsseldorf, Germany.

Drgas-Burchardt, E. and E. Lazuka, 2006. On chromatic polynomials of hypergraphs. Electron. Notes Discrete Math., 24: 105-110.

Kashif, M., 2011. Chromatic Polynomials and Chromaticity of some Linear H-Hypergraphs. VDM Verlag Dr.Muller, Saarbrucken, Germany, ISBN-13:978-3639348231, Pages: 120.

Khalaf, A.M. and Y.H. Peng, 2009. A note on chromaticity of k-bridge graphs. Far East J. Math. Sci., 34: 353-359.

Khalaf, A.M. and Y.H. Peng, 2010. A family of chromatically unique 6-bridge graphs. Ars Comb., 94 : 211-220.

Tomescu, I., 1998. Chromatic coefficients of linear uniform hypergraphs. J. Comb. Theor. Ser. B., 72: 229-235.

Walter, M., 2009. Some results on chromatic polynomials of hypergraphs. Electron. J. Combinatorics, 16: 1-16.

West, D.B., 2005. Introduction to Graph Theory. 2nd Edn., PHI Learning, Delhi, India, ISBN:9788120321427, Pages: 588.

Zhao, H., 2009. Chromatic Polynomial of Hypergraphs. Qinghai Normal University, Qinghai Sheng, China. 